\title{
Distribución conocida y potencial de dos taxa del género Mimosa (Leguminosae) endémicos de México
}

\author{
Susana Adriana Montaño-Arias ${ }^{1 *}$, Sara Lucía Camargo-Ricalde ${ }^{1 *}$, Rosaura Grether $^{1}$ \\ \& David Díaz-Pontones ${ }^{2}$ \\ 1. Departamento de Biología, División de Ciencias Biológicas y de la Salud, Universidad Autónoma Metropolitana- \\ Iztapalapa, Apdo. Postal 55-535, Ciudad de México, México; arias_susan@hotmail.com, slcr@xanum.uam.mx, \\ rogg@xanum.uam.mx \\ 2. Departamento de Ciencias de la Salud, División de Ciencias Biológicas y de la Salud, Universidad Autónoma \\ Metropolitana-Iztapalapa, Apdo. Postal 55-535, Ciudad de México, México; dmdp@xanum.uam.mx \\ * Correspondencia
}

Recibido 08-VI-2017. Corregido 24-X-2017. Aceptado 22-XI-2017.

\begin{abstract}
Present and potential distribution of two Mimosa (Leguminosae) taxa endemic to Mexico. Mimosa aculeaticarpa var. aculeaticarpa and M. luisana are endemic to Mexico, and are considered as multipurpose plants, due to the diverse services they offer to ecosystems and to local people. Additionally, they are appreciated for their potential to restore tropical environments; hence, the objective of this study was to model the present and potential distribution of these taxa. In 2014, species registers were obtained from two databases (CONABIO and MEXU); each register was taxonomically, geographically and statistically validated. Once validated, the present and potential distribution of M. aculeaticarpa var. aculeaticarpa (based on 99 registers) and $M$. luisana (based on 50 registers) were obtained using the MAXENT algorithm. For both taxa, the present distribution was overlapped using the layers of: elevation, climate, soil, biogeographic provinces, and hydrologic basins. Mimosa aculeaticarpa var. aculeaticarpa showed a wide distribution in Mexico (16 states); whilst M. luisana was restricted to the states of Puebla and Oaxaca. M. aculeaticarpa var. aculeaticarpa establishes between 1900 and 2700 masl, and M. luisana between 500 and 1760 masl. Both species were established in arid and semiarid climates; however, M. aculeaticarpa var. aculeaticarpa can also be found in temperate and mesic climates. Moreover, both taxa are distributed in calcareous regosol soils; although, M. aculeaticarpa var. aculeaticarpa is also found in eutric regosol, chromic vertisol and haplic phaeozem. The distribution of $M$. aculeaticarpa var. aculeaticarpa includes eight biogeographic provinces and three hydrologic basins; whilst $M$. luisana was only located in three provinces and two hydrologic basins; both are present in the Eje Volcánico and Sierra Madre del Sur provinces. The potential distribution models are considered as excellent ones due to an AUC of 0.91 and 0.97 , respectively; these models indicated that the temperature and precipitation conditions would be suitable for the enlargement of their distribution. Likewise, these models can be considered an approach to the potential distribution knowlegment of the Mexican mimosas. Nevertheless, it is important to note that the models are static and do not take into account any biotic interaction; therefore, their relationship with reality can vary. Thus, it is recommended to analyze the models through different climate change and land use scenarios. Rev. Biol. Trop. 66(1): 321-335. Epub 2018 March 01.
\end{abstract}

Key words: conservation; distribution; Leguminosae; MAXENT; Mexico; restoration.

El conocimiento de la distribución geográfica de los taxa proporciona información complementaria a la provista por estudios biológicos, ecológicos y sistemáticos, entre otros (Corsi, De Leeuw, \& Skidmore, 2000; Hernandez, Graham, Master, \& Albert, 2006) y es indispensable para el entendimiento de los procesos y patrones de la biodiversidad (Rushton, Ormerod, \& Kerby, 2004; Wisz et al., 2008), el efecto del cambio climático (Jeschke \& Strayer, 2008; Hole et al., 2009) y de los sitios con potencial para la reintroducción 
de taxa o para su posible restauración ambiental (Kumar \& Stohlgren, 2009).

La sobreexplotación de la biodiversidad y las fluctuaciones en los parámetros climáticos son los principales factores a los que se les atribuye la pérdida de los taxa (Cushman, 2006; Maxted \& Kell, 2009), por lo que se requiere de la búsqueda de estrategias que permitan la conservación de la biodiversidad. De acuerdo con Alsos, Alm, Normand y Brochmann (2009), para conservar un taxon se requiere conocer su respuesta a diferentes condiciones climáticas $\mathrm{y}$, por lo tanto, su capacidad de adaptación debida a los cambios en su entorno.

Se han propuesto diversos algoritmos basados en el concepto de nicho ecológico (nicho fundamental) establecido por Hutchinson (1957). Este concepto considera a los factores bióticos y abióticos necesarios para que las poblaciones de un taxon puedan sobrevivir indefinidamente sin la necesidad de migrar (Grinnell, 1917; Hutchinson, 1944). Sin embargo, en muchos casos los taxa no ocupan en su totalidad este nicho, por lo que el espacio utilizado se llama nicho realizado (Soberón \& Peterson, 2005).

Algunos de los algoritmos para modelar la distribución de los taxa son: el perfil Bioclimático, BIOCLIM (Nix, 1986); Algoritmo Genético, GARP (Stockwell \& Peters, 1999); y el de Máxima Entropía, MAXENT (Phillips, Dudík, \& Schapire, 2004), que están basados en datos de ocurrencia y/o ausencia, que permiten conocer la distribución de los taxa y han sido considerados herramientas fundamentales en la conservación y la ecología del paisaje (Peterson \& Cohoon, 1999). De acuerdo con varios autores, el algoritmo MAXENT es considerado como uno de los mejores modelos para predecir la distribución de los taxa (Elith et al., 2006; Ramírez-Villegas, Khoury, Jarvis, Debouck, \& Guarino, 2010; Herrera, 2012), ya que permite interacciones entre las variables, facilita investigar el efecto de cada variable (Mateo, 2008), y funciona con tamaños de muestra pequeños (Hernández et al., 2006).

Es importante mencionar que los trabajos sobre el modelado de la distribución de taxa mexicanos son escasos (Yberri, 2009). En este sentido, los taxa de la familia Leguminosae, una de las seis familias más diversas y mejor representadas en el país (Rzedowski, 1991; Villaseñor, 2016), están presentes en todos los tipos de vegetación y la mayoría son relevantes económicamente, por lo que es importante revisar su distribución conocida y estimar su distribución potencial.

A nivel mundial, los estudios que determinan la distribución conocida y/o potencial de los taxa de leguminosas son escasos, existen dos en África; uno enfocado en diferentes especies de Caesalpinoideae como Anthonotha gilletii (De Wild.) J. Léonard, Crudia harmsiana De Wild., C. laurentii De Wild., Cryptosepalum katangense (De Wild.) J. Léonard., Dialium pentandrum Steyaert, Leonardoxa romii (De Wild.) Aubrév. y Pseudomacrolobium mengei (De Wild.) Hauman (Ndayishimiye et al., 2012), y otro presentado por de la Estrella, Mateo, Wieringa, Mackinder y Muñoz (2012), quienes estudiaron los patrones de distribución de 185 especies de leguminosas. Otro estudio se realizó en Sudamérica, enfocado en diferentes especies, entre ellas Amburana cearensis A. C. Smith de la subfamilia Papilionoideae (Pacheco, Malizia, \& Cayuela, 2010); todos ellos se basaron en el algoritmo MAXENT. Para México, los trabajos son escasos, el primero fue llevado a cabo por Guevara-Escobar et al. (2008), quienes determinaron la distribución potencial de una selección de taxa arbustivos de leguminosas del bosque tropical caducifolio en el Altiplano Mexicano, y determinaron que Pithecellobium dulce (Roxb.) Benth., Calliandra eriophylla Benth. y C. formosa (Kunth) Benth. tienen una mayor área de distribución potencial en el Bajío; mientras que la menor área, pero con alta probabilidad de distribución, se registró para Prosopis laevigata (Willd.) M. C. Johnst., Albizia plurijuga (Standl.) Britt. et Rose y Leucaena leucocephala (Lam.) de Wit. El segundo trabajo está enfocado en aspectos filogeográficos del complejo Caesalpinia hintonii (Sotuyo et al., 2010), y el tercero fue llevado a cabo por Camargo-Ricalde (2015), quien determinó 
y analizó la distribución conocida y potencial de varios taxa multipropósito de los géneros Mimosa L. (25 taxa) y Prosopis L. (nueve taxa) (Leguminosae-Mimosoideae) presentes en el territorio mexicano.

En el caso particular de Mimosa, este es un género con distribución pantropical. En México, es el género más diverso de la subfamilia Mimosoideae; actualmente, se conocen ca. 104 especies (Grether, Camargo-Ricalde, MartínezBernal, Montaño-Arias, \& Fraile, 2015), por lo que se le considera el segundo lugar de diversificación, después de Brasil (Grether, CamargoRicalde, \& Martínez-Bernal, 1996). Debido a la importancia biológica y ecológica de los taxa de Mimosa en ambientes tropicales, éste ha sido objeto de múltiples estudios (sistemáticos, anatómicos, ecológicos y etnobotánicos, entre otros). Aunque se ha generado información significativa de diversas especies del género, el conocimiento relacionado con su distribución es parcial y está basado en trabajos florísticos (Martínez-Bernal \& Grether, 2006; Grether, Martínez-Bernal, \& Camargo-Ricalde, 2007; Martínez-Bernal, Grether, \& González-Amaro, 2008), por lo que es necesario generar información sobre su distribución potencial.

Por lo anterior, el objetivo de este estudio fue determinar la distribución conocida y potencial de Mimosa aculeaticarpa Ortega var. aculeaticarpa y M. luisana Brandegee, ambas endémicas de México, y cuya conservación es prioritaria, debido a que son taxa multipropósito, ya que brindan múltiples servicios a los ecosistemas y a los pobladores locales en donde se establecen; además de que se consideran útiles para la restauración de ambientes tropicales (Dhillion, Aguilar-Støen, \& Camargo-Ricalde, 2004; Martínez-Pérez, Orozco-Segovia, \& Mantorell, 2006; Pavón, Ballato-Santos, \& Pérez-Pérez, 2011).

\section{MATERIALES Y MÉTODOS}

Selección de los taxa: Se seleccionó a Mimosa aculeaticarpa var. aculeaticarpa con distribución amplia en el país, ya que habita en climas secos como templados (Grether et al., 2007; Martínez-Bernal et al., 2008) y a $M$. luisana que es un taxon restringido al Valle de Tehuacán-Cuicatlán, Puebla y Oaxaca, y sólo se establece en climas secos (Martínez-Bernal \& Grether, 2006).

Obtención de datos: En el año 2014, se accedió a la base de datos de Leguminosas proporcionada por la Comisión Nacional para el Conocimiento y Uso de la Biodiversidad (CONABIO) y a la base de datos del Herbario Nacional (MEXU) para la revisión y extracción de los registros de ambos taxa. Este estudio tuvo una duración de 21 meses; inició en octubre de 2014 y concluyó en junio de 2016; es relevante señalar que la depuración de las bases de datos y la revisión de ejemplares de herbario duró $c a$. de 18 meses.

Por taxon, se elaboró una base de datos, a la cual se le incorporaron registros de consultas de herbarios (siglas de los herbarios de acuerdo con Thiers, 2016): Herbario Nacional de México (MEXU), Instituto de Biología, Universidad Nacional Autónoma de México; Herbario Metropolitano (UAMIZ), Universidad Autónoma Metropolitana-Iztapalapa; Herbario del Centro Regional del Bajío (IEB), Instituto de Ecología, A.C., Pátzcuaro, Michoacán, y del Herbario de la Facultad de Ciencias Naturales (QMEX), Universidad Autónoma de Querétaro.

Depuración y validación de datos: La base de datos de cada taxon, se validó taxonómicamente, corrigiendo errores de omisión, tipografía, contexto, redundancia, convención y congruencia. Para poder realizar las correcciones, se establecieron criterios taxonómicos y geográficos (depuración geográfica) mínimos a cumplir por cada registro de la base de datos (Cuadro 1), de manera que la base de datos de M. aculeaticarpa var. aculeaticarpa, contiene registros de ejemplares recolectados entre 1932 y 2011, y la de M. luisana entre 1908 y 2014 .

La depuración geográfica consistió en la validación de las coordenadas geográficas de cada registro con el programa ArcMap ver. 10.2.2 (ESRI, 2014), la cobertura de municipios 
CUADRO 1

Criterios taxonómicos y geográficos considerados para cada registro de la base de datos

TABLE 1

Taxonomic and geographic criteria considered for each record of the database

\begin{tabular}{ll}
\multicolumn{1}{c}{ Criterios Taxonómicos } \\
$\begin{array}{ll}\text { De la base de CONABIO, sólo se tomaron } \\
\text { los registros que cuentan con el ejemplar de } \\
\text { herbario. }\end{array}$ & $\begin{array}{l}\text { Revisión de áreas de distribución a priori (municipios, estados) por } \\
\text { taxon, definidas con base en la experiencia del especialista y en } \\
\text { publicaciones taxonómicas y florísticas. }\end{array}$ \\
$\begin{array}{l}\text { En el caso de } M \text {. aculeaticarpa var. } \\
\text { aculeaticarpa, si el registro no presentaba la } \\
\text { variedad, éste se descartó. }\end{array}$ & $\begin{array}{l}\text { Selección de un ejemplar por taxon, cuando existen varios registros de la } \\
\text { misma localidad. }\end{array}$ \\
$\begin{array}{l}\text { Revisión del nombre de los taxa (ortografía y } \\
\text { sinonimia). }\end{array}$ & $\begin{array}{l}\text { Que los registros coincidieran con los rangos altitudinales en el que se } \\
\text { desarrolla cada taxon. }\end{array}$ \\
$\begin{array}{l}\text { Experiencia del especialista que determinó el } \\
\text { ejemplar. }\end{array}$ & $\begin{array}{l}\text { Que las coordenadas geográficas coincidieran con el sitio de colecta del } \\
\text { taxon. } \\
\text { Que los registros georreferenciados correspondieran a localidades en } \\
\text { donde previamente había sido observado (colectado) el taxon y en donde } \\
\text { se presentan las condiciones para que los taxa puedan existir. }\end{array}$ \\
\hline
\end{tabular}

(INEGI, 2005) y el nomenclator de CONABIO (2014). Cuando se detectaron municipios equivocados, se corrigieron y se contabilizaron como puntos validados.

La especialista del género, Rosaura Grether, de la Universidad Autónoma Metropolitana (UAM), Unidad Iztapalapa, validó la distribución conocida del taxon en una coordenada proporcionada; de esta forma, se determinó a priori un posible patrón de distribución de cada taxon, de manera que aquellos registros que no se ajustaron al mismo, se consideraron como localidades erróneas. Las bases de datos están resguardadas en el laboratorio de Biosistemática de Leguminosas, del Departamento de Biología, de la UAM-Iztapalapa.

Distribución conocida: Los datos depurados se posicionaron sobre las siguientes capas: i) elevación, ii) climas (García-CONABIO, 1998), iii) suelos (INIFAP, 1995), iv) provincias biogeográficas (CONABIO, 1997), y v) cuencas hidrológicas (CNA, 1998), obtenidas del portal de geoinformación de la CONABIO (2011), utilizando el programa ArcMap ver. 10.2.2. La clasificación utilizada es la propuesta por CONABIO (2011).
Distribución potencial: Se modeló con base en una aproximación de nicho ecológico (Peterson et al., 2011), usando el algoritmo de máxima entropía MAXENT (Phillips, Anderson, \& Schapire, 2006).

Validación estadística: Después de la validación taxonómica y geográfica, los datos se validaron estadísticamente con el programa SPSS (2012, Statistics versión 21, www. ibm.com.analytics/spss). Se identificaron los datos atípicos mediante diagramas de caja (box $p l o t)$, producto de un cruce entre los registros (localidades únicas) y las coberturas ambientales. Una vez eliminados los datos atípicos, los datos validados se utilizaron para crear el perfil bioclimático de cada taxon mediante estadística descriptiva.

Para la modelación de la distribución potencial, se utilizaron las coberturas obtenidas de Worldclim (http://www.worldclim. org/ - Hijmans, Cameron, Parra, Jones, \& Jarvis, 2005 - Cuadro 2), recortadas para México. La selección de variables climáticas se realizó generando un modelo en MAXENT, con las 19 variables climáticas y mediante la gráfica de Jacknife que crea este algoritmo. 
CUADRO 2

Variables climáticas de Worldclim, utilizadas por MAXENT, para generar los mapas de distribución potencial de los dos taxa de Mimosa estudiados

TABLE 2

Worldclim climatic variables, used by MAXENT, to generate maps of potential distribution of the two Mimosa taxa studied

\begin{tabular}{cll} 
No. & Abr. & Variable Climática \\
1 & TPA & Temperatura promedio anual $\left({ }^{\circ} \mathrm{C}\right)$ \\
2 & ODT & Oscilación diurna de la temperatura $\left({ }^{\circ} \mathrm{C}\right)$ \\
3 & OAT & Oscilación anual de la temperatura $\left({ }^{\circ} \mathrm{C}\right)($ cociente entre parámetros 4 y 5$)$ \\
4 & TPPC & Temperatura máxima promedio del período más cálido $\left({ }^{\circ} \mathrm{C}\right)$ \\
5 & TPPF & Temperatura mínima promedio del período más frío $\left({ }^{\circ} \mathrm{C}\right)$ \\
6 & TCLL & Temperatura promedio del cuatrimestre más lluvioso $\left({ }^{\circ} \mathrm{C}\right)$ \\
7 & TCS & Temperatura promedio del cuatrimestre más seco $\left({ }^{\circ} \mathrm{C}\right)$ \\
8 & TCC & Temperatura promedio del cuatrimestre más cálido $\left({ }^{\circ} \mathrm{C}\right)$ \\
9 & TCF & Temperatura promedio del cuatrimestre más frío $\left({ }^{\circ} \mathrm{C}\right)$ \\
10 & ISO & Isotermalidad $\left({ }^{\circ} \mathrm{C}\right)($ cociente entre parámetros 2 y 7$)$ \\
11 & EDT & Estacionalidad de la temperatura $($ coeficiente de variación, en \%) \\
12 & EP & Estacionalidad de la precipitación $($ coeficiente de variación, en \%) \\
13 & PA & Precipitación anual (mm) \\
14 & PPLL & Precipitación promedio del período más lluvioso $(\mathrm{mm})$ \\
15 & PPS & Precipitación promedio del período más seco $(\mathrm{mm})$ \\
16 & PCLL & Precipitación promedio del cuatrimestre más lluvioso $(\mathrm{mm})$ \\
17 & PCS & Precipitación promedio del cuatrimestre más seco $(\mathrm{mm})$ \\
18 & PCC & Precipitación promedio del cuatrimestre más cálido $(\mathrm{mm})$ \\
19 & PCF & Precipitación promedio del cuatrimestre más frío $(\mathrm{mm})$ \\
\hline
\end{tabular}

Igualmente, se realizó un análisis de correlación Pearson debido a que el modelo podría sesgarse por la correlación entre variables. La correlación se llevó a cabo al obtener un cruce de los registros de cada taxon con las 19 variables climáticas.

Criterio de selección de la región de referencia (movilidad $=\mathbf{M})$ : El criterio para modelar la distribución de los taxa fue mediante su patrón de distribución geográfica. La región M fue determinada considerando límites geográficos naturales, y se empleó la cobertura de cuencas hidrológicas de México (CNA, 1998).

Evaluación de los modelos: Se utilizó el área bajo la curva (AUC, por sus siglas en inglés), que ha sido aceptado como un método estándar para evaluar la exactitud de los modelos de distribución (Benito de Pando \&
Peñas de Giles, 2007; Lobo, Jiménez-Valverde, \& Real, 2008). El valor utilizado del AUC fue el generado por el algoritmo MAXENT y la categoría del modelo se basó en Burgueño, García-Bastos y González-Buitrago (1995), y Menzaour (2005).

\section{RESULTADOS}

Depuración y validación de datos: En las bases de datos consultadas se detectaron problemas taxonómicos y geográficos. Los problemas taxonómicos fueron principalmente la determinación incorrecta de algunos ejemplares de herbario o a casos de sinonimia.

En la base de datos de la CONABIO, se encontraron 281 registros de $M$. aculeaticarpa var. aculeaticarpa, y 72 de M. luisana. En el caso de M. aculeaticarpa var. aculeaticarpa, 221 registros fueron eliminados 
por no especificar la variedad (aculeaticarpa o desmanthocarpa). Asimismo, 17 registros se descartaron por hacer referencia a taxa distintos; además, 11 registros de $M$. aculeaticarpa var. aculeaticarpa y cuatro de $M$. luisana fueron retirados porque se ubicaron fuera de sus patrones de distribución.

También, se detectó que dos registros de M. aculeaticarpa var. aculeaticarpa y cuatro de $M$. luisana tenían municipios equivocados, éstos se corrigieron y se tomaron como datos validados. Asimismo, ambos taxa presentaron localidades repetidas; es decir, que habían sido recolectadas varias veces en el mismo lugar, por lo que sólo se conservó un registro y los demás fueron descartados (cuatro y 27 , respectivamente).

Adición de registros: Se elaboró una base de datos para M. aculeaticarpa var. aculeaticarpa con 40 registros validados provenientes de la CONABIO; se le adicionaron 59 registros más, provenientes de la revisión de las colecciones de los herbarios IEB y MEXU, lo que permitió elaborar el patrón de distribución geográfica del taxon. Respecto a la base de datos de $M$. luisana, ésta tuvo 38 registros validados provenientes de la CONABIO; y se le adicionaron 12 registros del IEB.

Distribución conocida: Los resultados indican que $M$. aculeaticarpa var. aculeaticarpa se encuentra en 16 estados de la República Mexicana (Fig. 1A). Los estados con el mayor número de registros son: Michoacán, Guanajuato, Oaxaca y Querétaro; mientras que Aguascalientes, Zacatecas, Guerrero, Tlaxcala y Veracruz sólo presentaron un registro. En el caso de $M$. luisana, el mayor número de colectas ha sido realizado en el estado de Puebla (Fig. 1B).

El análisis de la capa de elevación, mostró que $M$. aculeaticarpa var. aculeaticarpa puede localizarse desde los 0 hasta los $2500 \mathrm{msnm}$, y M. luisana de 0 a $2000 \mathrm{msnm}$. En M. aculeaticarpa var. aculeaticarpa, el mayor número de localidades se ubicó entre 1500 y 2000 msnm
(Fig. 1C), y en M. luisana, 15 localidades se ubicaron entre 500 y $1000 \mathrm{msnm}$, y otras 14 se localizaron entre 1500 y $2000 \mathrm{msnm}$ (Fig. 1D). Sin embargo, al tomar directamente los datos originales (etiquetas de herbario), los rangos altitudinales son más estrechos, ya que $M$. aculeaticarpa var. aculeaticarpa se distribuye entre los 1900 y $2700 \mathrm{msnm}$, y M. luisana entre los 500 y $1760 \mathrm{msnm}$.

Por otro lado, la sobre-posición de las localidades de ambos taxa con la capa de climas, indicó que el mayor número de registros de $M$. aculeaticarpa var. aculeaticarpa se encuentra en climas secos; así como en áridos, semiáridos, templados y mésicos (Fig. 1E); mientras que la mayoría de las localidades de M. luisana están ubicadas en climas áridos (Fig. 1F).

Además, las colectas de $M$. aculeaticarpa var. aculeaticarpa se han realizado en suelos de tipo regosol éutrico, seguido del vertisol crómico, regosol calcárico y del feozem háplico (Fig. 2A); mientras que M. luisana predomina en suelos de tipo regosol calcárico (Fig. 2B).

Los resultados muestran que la distribución de M. aculeaticarpa var. aculeaticarpa abarca ocho provincias biogeográficas: 1) Altiplano Norte, 2) Altiplano Sur, 3) Sierra Madre Oriental, 4) Tamaulipeca, 5) Eje Volcánico, 6) Depresión del Balsas, 7) Sierra Madre del Sur, y 8) Golfo de México (Fig. 2C). Cabe mencionar que el Eje Volcánico es la provincia con el mayor número de localidades, seguido del Altiplano Sur. En el caso de M. luisana, se observó que se distribuye en tres provincias biogeográficas: 1) Eje Volcánico, 2) Oaxaca, y 3) Sierra Madre del Sur (Fig. 2D).

Por otra parte, el análisis de la capa de cuencas mostró que el mayor número de colectas de M. aculeaticarpa var. aculeaticarpa se han realizado en la Cuenca del Río Moctezuma, en la Cuenca del Lago de Pátzcuaro-Cuitzeo y el Lago de Yuridia (Fig. 2E); mientras que las localidades de $M$. luisana se encuentran asociadas a la Cuenca del Río Papaloapan y la del Río Atoyac; aunque, en la primera es en donde está la mayor parte de los datos de presencia (Fig. 2F). 


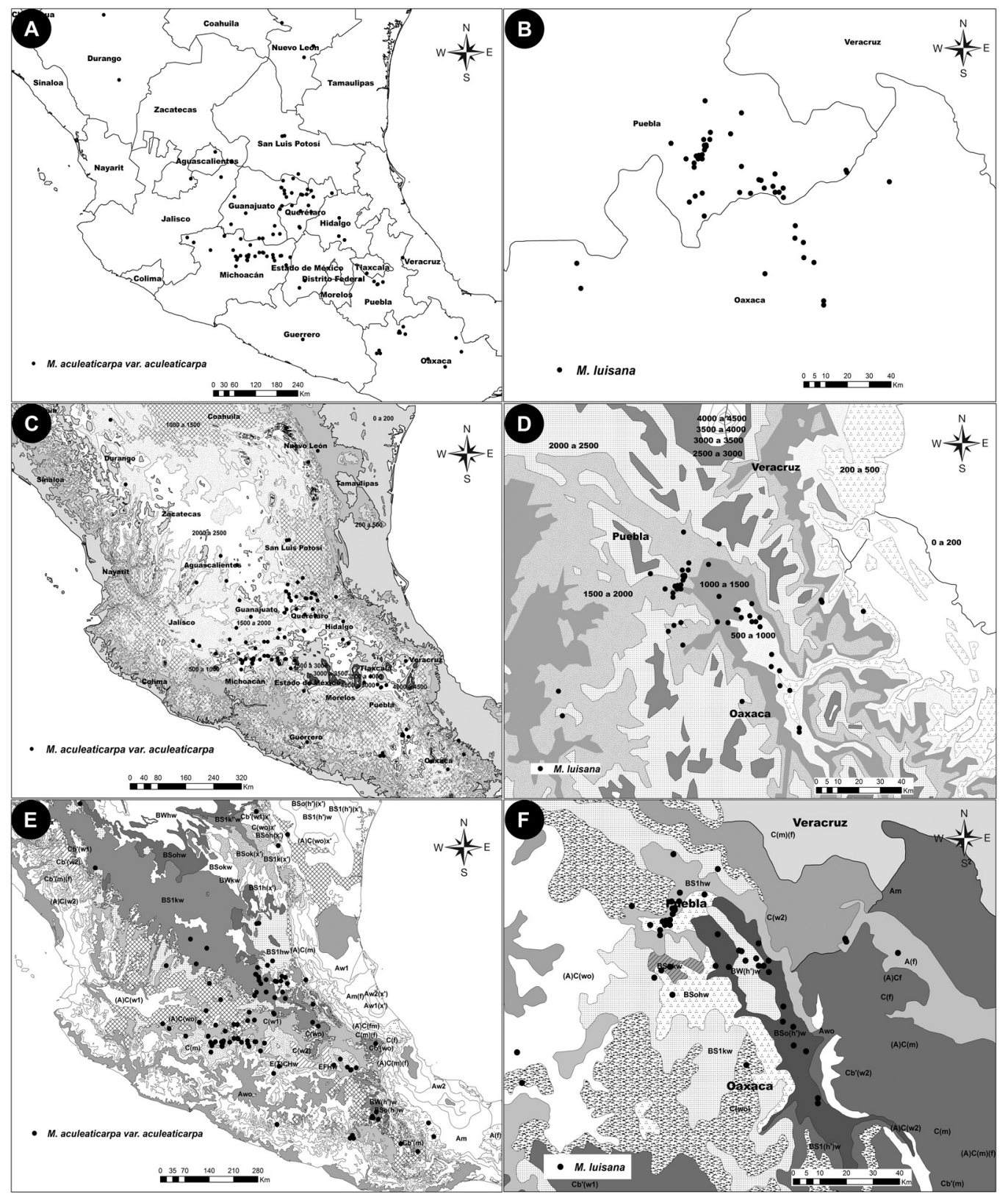

Fig. 1. Distribución conocida (localidades en puntos negros) de M. aculeaticarpa var. aculeaticarpa (A, C, E) y M. luisana (B, D, F), posicionadas sobre las capas de: Estados, escala 1:1 000000 (A, B); Elevación (msnm), escala 1:4 000000 (C, D); y Climas, escala 1:1000 000 (E, F).

Fig. 1. Present distribution (localities in black dots) of M. aculeaticarpa var. aculeaticarpa (A, C, E) and M. luisana (B, D, F), positioned on the layers: States, scale 1:1 000000 (A, B); Elevation (mosl), scale 1:4 000000 (C, D); and Climates, scale 1:1000 $000(\mathrm{E}, \mathrm{F})$. 


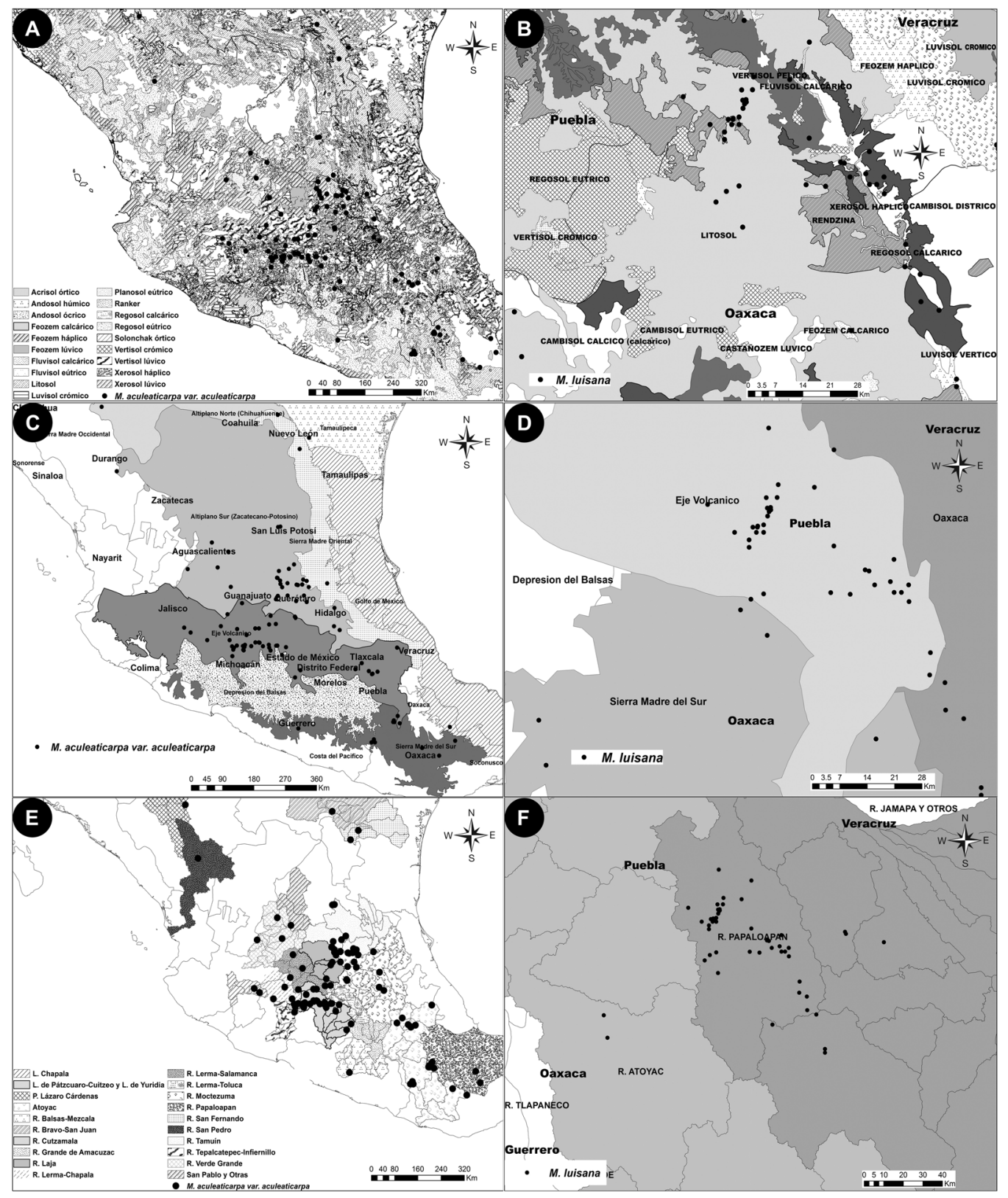

Fig. 2. Distribución conocida (localidades en puntos negros) de M. aculeaticarpa var. aculeaticarpa (A, C, E) y M. luisana (B, D, F), posicionadas sobre las capas de: Suelos, escalas 1:250 000 y 1:1 000000 (A, B); Provincias biogeográficas, escala 1:4 000000 (C, D); y Cuencas hidrológicas, escala 1:250 000 (E, F).

Fig. 2. Present distribution (localities in black dots) of M. aculeaticarpa var. aculeaticarpa (A, C, E) and M. luisana (B, D, F), positioned on the layers: Soils, scales 1:250 000 and 1:1 000000 (A, B); Biogeographic provinces, scale 1:4 000 000 (C, D); and Watersheds, scale 1:250 000 (E, F). 
Distribución potencial: El perfil bioclimático generado para $M$. aculeaticarpa var. aculeaticarpa muestra que este taxon puede habitar en lugares donde la temperatura promedio anual sea de $17.9^{\circ} \mathrm{C}$, con una precipitación promedio anual de $959 \mathrm{~mm}$; mientras que $M$. luisana puede establecerse en lugares con una temperatura promedio anual de $21.1{ }^{\circ} \mathrm{C}$ y con una precipitación promedio anual de $563.2 \mathrm{~mm}$.

El Jacknife generado para el modelo de $M$. aculeaticarpa var. aculeaticarpa, mostró que las variables Estacionalidad de la temperatura (EDT, $18.5 \%$ ) y Temperatura promedio del cuatrimestre más cálido (TCC, $54.4 \%$ ) son las que aportan mayor información al modelo. Asimismo, el Jacknife indicó que el modelo se sesgaría si no se consideraban las variables EDT y Precipitación promedio del cuatrimestre más frío (PCF). La información proporcionada por el Jacknife se complementó con el análisis de correlación de Pearson y, éste último, evidenció que EDT y PCF son las únicas variables independientes; por lo que éstas se utilizaron para generar el modelo final.

En el caso de M. luisana, el análisis Jacknife mostró que las variables EDT (31.5\%) y PCF (45.7\%) son las variables que aportan mayor información al modelo; además de mostrar que la variable EDT debería ser considerada para no sesgar el modelo. Por lo anterior, el modelo final se generó utilizando las variables EDT y PCF.

Los modelos de ambos taxa se consideran excelentes por presentar un AUC de $0.91 \mathrm{y}$ 0.97 , respectivamente, lo que indica una predicción con alta exactitud.

El modelo de distribución potencial de $M$. aculeaticarpa var. aculeaticarpa, sin considerar su movilidad (M), sugiere que existen sitios (penínsulas de Baja California y de Yucatán) con las condiciones adecuadas para que este taxon amplíe su distribución, desde el centro del país hacia la región del Altiplano Sur (Zacatecano-Potosino); también hacia el noroeste del Eje Volcánico y hacia la parte sur de la Sierra Madre del Sur (Fig. 3A). Igualmente, el modelo de distribución potencial de $M$. luisana indica que, actualmente, existen las condiciones para que este taxon también amplíe su distribución hacia la región noreste del Eje Volcánico, las regiones suroeste y sureste de la Sierra Madre del Sur, y abarcar, en su totalidad, la provincia biogeográfica de Oaxaca y extenderse hacia una pequeña porción del oeste de la provincia del Golfo de México (Fig. 3B). La predicción indica que este taxon podría ampliar su distribución en la región oeste del Golfo de México, región en la cual, hasta ahora, sólo cuenta con un único registro. No obstante, cuando se genera la región $\mathrm{M}$ de $M$. aculeaticarpa var. aculeaticarpa, se observa que no es posible localizarla en la Península de Baja California, ni en la de Yucatán, ni en el estado de Chiapas. Además, muestra que la distribución de este taxon podría extenderse hacia el Altiplano Zacatecano-Potosino, región que cuenta con pocos registros de colecta; de la misma forma, se podría ampliar hacia la parte oeste del Eje Volcánico y el suroeste del Altiplano Norte (Chihuahuense) (Fig. 3C). Asimismo, cuando se genera el modelo reconociendo la movilidad de $M$. luisana, se detecta que éste no llega al centro del estado de Oaxaca y, menos aún, al estado de Chiapas. Además, existen otros puntos donde están presentes las condiciones climáticas para el establecimiento del taxon, pero existen límites geográficos que impiden su ocurrencia en esos sitios (Fig. 3D).

\section{DISCUSIÓN}

Respecto a la distribución conocida, los resultados muestran una variación en cuanto al número de registros obtenidos por taxon, esto debido a que $M$. aculeaticarpa var. aculeaticarpa presenta una distribución amplia, $\mathrm{y}$ $M$. luisana tiene una distribución restringida. La primera está presente en 16 estados de la República, lo que concuerda con lo reportado por Grether et al. (2007) y Martínez-Bernal et al. (2008). En el caso de la distribución de $M$. luisana, ésta coincide en que sólo se establece en los estados de Puebla y Oaxaca, como lo reportan Martínez-Bernal y Grether (2006).

Asimismo, los mapas de la distribución conocida indican, con precisión, los sitios 


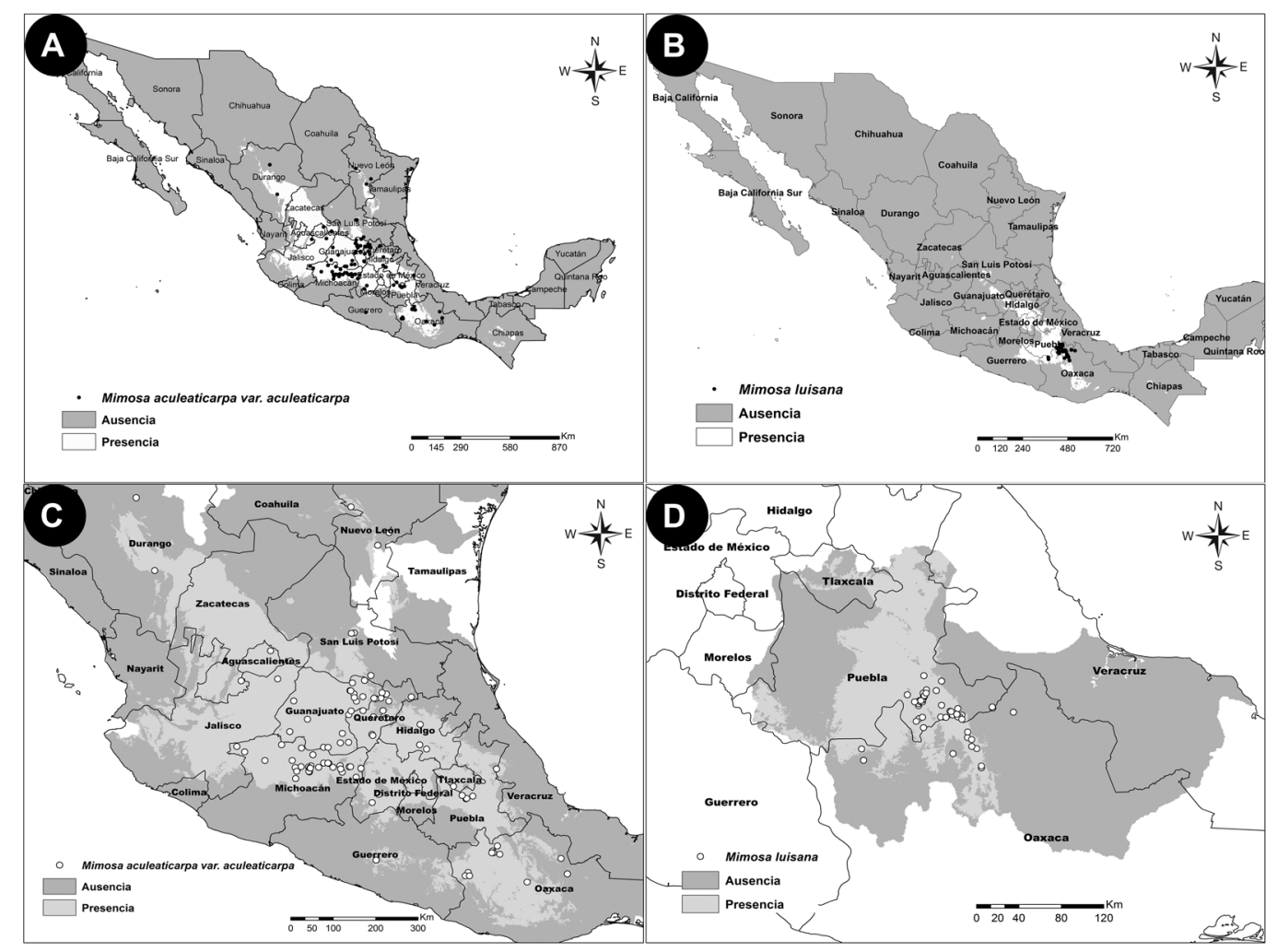

Fig. 3. Distribución potencial sin considerar la movilidad del taxon (localidades en puntos negros y blancos) de $M$. aculeaticarpa var. aculeaticarpa (A) y M. luisana (B). Distribución potencial considerando la movilidad de M. aculeaticarpa var. aculeaticarpa (C) y M. luisana (D); escala 1:1000 000 .

Fig. 3. Potential distribution without considering the mobility of the taxon of M. aculeaticarpa var. aculeaticarpa (A) and M. luisana (B). Potential distribution considering the mobility of M. aculeaticarpa var. aculeaticarpa (C) and M. luisana (D); scale 1:1000 000 .

en donde se tienen más registros (colectas) para cada taxon (M. aculeaticarpa var. aculeaticarpa en Michoacán y M. luisana en Puebla), lo que sugiere un sesgo en los métodos, al momento de generar los modelos predictivos (Phillips et al., 2009); sin embargo, los modelos generan una distribución hipotética que, de acuerdo con Trotta-Moreu, Lobo y CabreroSañudo (2008), puede ser utilizada tanto en la conservación como en la biogeografía de los taxa; además de ser una herramienta útil para la planeación de nuevas colectas.

La modelación de este tipo de distribución muestra que la mayoría de los registros, en ambos taxa, se localizan en altitudes entre 1500 a $2000 \mathrm{msnm}$; este rango altitudinal es congruente con lo registrado por Grether et al. (2015).

Respecto a los climas, ambos taxa se encuentran en sitios con climas secos, áridos y semiáridos; aunque, M. aculeaticarpa var. aculeaticarpa, también se encuentra en sitios templados y mésicos, lo que coincide con lo reportado por Martínez-Bernal y Grether (2006), Grether et al. (2007) y Martínez-Bernal et al. (2008).

Por otro lado, algunos autores como Lindenmayer, Mackey y Nix (1996) y Peterson, Egbert, Sánchez-Cordero y Price (2000), consideran que para generar los modelos de distribución potencial es necesario contar con, al menos, 50 registros para poder predecir 
la distribución de los taxa; sin embargo, existen estudios que han utilizado menos registros (12 o 23) y los modelos han sido considerados con un alto nivel de predicción (Villaseñor \& Téllez-Valdez, 2004; Palacios, 2010). Cabe mencionar que, en este estudio, sólo $M$. aculeaticarpa var. aculeaticarpa contó con más de 50 registros, pero los modelos obtenidos para ambos taxa, tuvieron una excelente predicción.

Además, para ambos taxa, los modelos de distribución potencial coinciden en gran parte con su distribución conocida, lo que muestra un bajo error de omisión (Contreras-Medina, Luna-Vega, \& Ríos-Muñoz, 2010). Igualmente, dichos modelos presentan un valor AUC alto, por lo que se pueden considerar robustos (Marmion, Parviainen, Luoto, Heikkinen, \& Thuiller, 2009; Marini, Barbet-Massin, Martínez, Prestes, \& Jiguet, 2010). Sin embargo, los resultados señalan que el nivel de predicción del modelo de distribución potencial de $M$. aculeaticarpa var. aculeaticarpa fue inferior $(\mathrm{AUC}=0.91)$ que el de $M$. luisana $(\mathrm{AUC}=$ 0.97), lo que sugiere que el modelado de un taxon con amplia distribución, es menos preciso que uno correspondiente a un taxon con distribución restringida, lo que coincide con lo reportado por Hernández et al. (2006), Naoki, Gómez, López, Meneses y Vargas, (2006) y Guevara-Escobar et al. (2008).

En ese mismo sentido, algunos autores como Engler, Guisan y Rechsteiner (2004), y Chefaoui y Lobo (2008) consideran que los modelos basados solamente en datos de presencia, pueden sobreestimar la distribución de los taxa. En este caso, aunque el modelo de distribución potencial de $M$. aculeaticarpa var. aculeaticarpa es considerado un modelo excelente, podría indicar una sobreestimación de su distribución (algunas partes de la Península de Baja California y Península de Yucatán). De igual manera, sucede con el modelo de $M$. luisana, ya que predice que este taxon puede ampliar su distribución hacia las regiones centro y suroeste de Oaxaca, y centro de Chiapas, debido a que existen las condiciones de temperatura y precipitación adecuadas para que se desarrolle. Al respecto, existen estudios que sugieren que para robustecer los modelos predictivos es necesario incorporar otros factores como la topografía (Naoki et al., 2006; Aalto \& Luoto, 2014) y considerar los datos históricos de los taxa (Escalante, 2003).

Aunque la delimitación de la región $\mathrm{M}$ de los taxa es un tema poco tratado en la literatura, probablemente es una forma más para robustecer los modelos. De acuerdo con Brown (1995), la distribución de un taxon está determinada por la interacción entre su ecología y su historia evolutiva, por lo que para realizar predicciones de su distribución, se deben considerar sus límites geográficos, ya que ello permite reconocer sus límites de dispersión (Soberón \& Peterson, 2005).

Dado lo anterior, la validación de los modelos es un tema controversial; si bien el AUC es un método aceptado (Benito de Pando $\&$ Peñas de Giles, 2007), se requiere de la validación del modelo por métodos de muestreo en campo (Contreras-Medina et al., 2010), ya que los algoritmos no consideran las interacciones bióticas que pueden afectar la distribución de los taxa (Naoki et al., 2006; Palacios, 2010). Sin embargo, en este caso particular (i.e. experiencia taxonómica y florística del género Mimosa en México), los modelos generados en este trabajo pueden considerarse como una aproximación al conocimiento de la distribución potencial de los taxa estudiados.

Finalmente, desde una perspectiva de cambio climático, los modelos de distribución potencial de ambos taxa, sugieren una reducción y una ampliación de su área de distribución; ya que, de acuerdo con Gómez-Díaz, Monterroso-Rivas y Tinoco-Rueda (2007), los ecosistemas templados podrían reducirse y los secos ampliarse. Bajo esta premisa, M. aculeaticarpa var. aculeaticarpa podría reducir su distribución; mientras que $M$. luisana la podría ampliar. No obstante, se sugiere profundizar este estudio abarcando diferentes escenarios de cambio climático y de uso de suelo; ya que son de las posibles causas que pudieran afectar a los ecosistemas (Parmensan \& Yohe, 2003).

Considerando que Mimosa es un género pantropical y que México es el segundo centro 
de distribución de este género, los mapas de distribución conocida y potencial de sus taxa deben considerarse como una aproximación al conocimiento de las mimosas mexicanas.

Asimismo, este trabajo aporta datos e información sobre zonas del territorio nacional que no han podido ser estudiadas por diversas causas (P. ej. dificultad de acceso y seguridad, entre otras), los cuales podrían ser corroborados en el campo posteriormente; aunque, es importante considerar que los modelos son estáticos, ya que no consideran las interacciones bióticas, por lo que su relación con la realidad puede variar.

Por último, aunque los modelos aquí presentados son una aproximación a la distribución conocida y potencial de los taxa estudiados, es importante incorporar otros aspectos (además de los climáticos) como los históricos y topográficos, así como los edáficos y el cambio de uso de suelo, para una mayor precisión en la predicción de los modelos.

\section{AGRADECIMIENTOS}

Al Doctorado en Ciencias Biológicas y de la Salud de la Universidad Autónoma Metropolitana y al Consejo Nacional de Ciencia y Tecnología (CONACYT) por la beca de doctorado (228993/211528) otorgada a SAMA.

\section{RESUMEN}

Mimosa aculeaticarpa var. aculeaticarpa y M. luisana son endémicas de México y consideradas plantas multipropósito, ya que ofrecen diversos servicios a los ecosistemas y pobladores en donde se establecen. Además, son valoradas por su potencial como restauradoras de ambientes tropicales, por lo que el objetivo de este estudio fue modelar su distribución conocida y potencial. En el año 2014, se obtuvieron registros de dos bases de datos (CONABIO y MEXU); cada resgistro fue validado taxonómica, geográfica y estadísticamente, una vez validados, se obtuvo la distribución conocida y potencial para $M$. aculeaticarpa var. aculeaticarpa (basada en 99 registros) y M. luisana (basada en 50 registros), utilizando el algoritmo MAXENT. La distribución conocida de ambos taxa se sobreposicionó en las capas de: elevación, clima, suelo, provincias biogeográficas y cuencas hidrológicas. Mimosa aculeaticarpa var. aculeaticarpa presenta amplia distribución en México (16 estados); mientras que $M$. luisana se encuentra restringida a los estados de Puebla y Oaxaca. M. aculeaticarpa var. aculeaticarpa se establece entre 1900 y $2700 \mathrm{msnm}$ y $M$. luisana entre 500 y $1760 \mathrm{msnm}$. Ambas se encuentran en climas áridos y semiáridos; sin embargo, M. aculeaticarpa var. aculeaticarpa también se puede encontrar en climas templados y mésicos. Asimismo, ambos taxa se distribuyen en suelos de tipo regosol calcárico; aunque, M. aculeaticarpa var. aculeaticarpa también está en regosol éutrico, vertisol crómico y feozem háplico. La distribución de $M$. aculeaticarpa var. aculeaticarpa abarca ocho provincias biogeográficas y tres cuencas hidrológicas; mientras que M. luisana se localiza en tres provincias y dos cuencas; ambas coinciden en las provincias del Eje Volcánico y la Sierra Madre del Sur. Los modelos de distribución potencial se consideran excelentes, ya que poseen un AUC de 0.91 y 0.97 , respectivamente. Los modelos indican que las condiciones de temperatura y precipitación son propicias para que ambos taxa pudieran ampliar su distribución. Igualmente, los modelos generados pueden considerarse como una aproximación al conocimiento de la distribución potencial de las mimosas mexicanas. Aunque, es importante considerar que los modelos son estáticos y no consideran a las interacciones bióticas, por lo que su relación con la realidad puede variar; por lo que se recomienda analizar los modelos mediante diferentes escenarios de cambio climático y de uso de suelo.

Palabras clave: conservación; distribución; Leguminosae; MAXENT; México; restauración.

\section{REFERENCIAS}

Aalto, J., \& Luoto, M. (2014). Integrating climate and local factors for geomorphological distribution models. Earth Surface Processes and Landforms, 39, 17291740. doi: 10.1002/esp.3554

Alsos, I. G., Alm, T., Normand, S., \& Brochmann, C. (2009). Past and future range shifts and loss of diversity in dwarf willow (Salix herbacea L.) inferred from genetics, fossils and modeling. Global Ecology and Biogeography, 18, 223-239. doi: 10.1111/j.1466-8238.2008.00439.x.

Benito de Pando, B., \& Peñas de Giles, J. (2007). Aplicación de modelos de distribución de especies a la conservación de la biodiversidad en el sureste de la Península Ibérica. GeoFocus, 7, 100-119.

Brown, J. H. (1995). Macroecology. Illinois, Estados Unidos: University of Chicago Press.

Burgueño, M. J., García-Bastos, L., \& González-Buitrago, J. M. (1995). Las curvas ROC en la evaluación de pruebas diagnósticas. Medicina Clínica, 104, 661-670. 
Camargo-Ricalde, S. L. (2015). Modelación de la distribución real y potencial de especies de dos géneros de Mimosoideae: Mimosa y Prosopis (Leguminosae) en México. Universidad Autónoma Metropolitana. Unidad Iztapalapa. Informe Final SNIB_CONABIO proyecto No. JM050. México, D.F.

Chefaoui, R. M., \& Lobo, J. M. (2008). Assessing the effects of pseudo-absences on predictive distribution model performance. Ecological Modelling, 210, 478486. doi:10.1016/j.ecolmodel.2007.08.010.

CNA (Comisión Nacional del Agua). (1998). Cuencas hidrológicas de la República Mexicana, escala 1:250000. Comisión Nacional para el Conocimiento y Uso de la Biodiversidad (CONABIO), México.

CONABIO (Comisión Nacional para el Conocimiento y Uso de la Biodiversidad). (1997). 'Provincias biogeográficas de México'. Escala 1:4 000 000. Comisión Nacional para el Conocimiento y Uso de la Biodiversidad, México, D. F.

CONABIO (Comisión Nacional para el Conocimiento y Uso de la Biodiversidad). (2011). Portal de geoinformación Sistema Nacional de Información sobre biodiversidad. Recuperado de http:/www.conabio. gob.mx

CONABIO (Comisión Nacional para el Conocimiento y Uso de la Biodiversidad). (2014). Localidades rurales y urbanas 2, 2010', escala: 1:1. Comisión Nacional para el Conocimiento y Uso de la Biodiversidad. Datos estadísticos del 2010, del Instituto Nacional de Estadística y Geografía (INEGI). México, D.F.

Contreras-Medina, R., Luna-Vega, I., \& Ríos-Muñoz, C. A. (2010). Distribución de Taxus globosa (Taxaceae) en México: Modelos ecológicos de nicho, efectos del cambio del uso de suelo y conservación. Revista Chilena de Historia Natural, 83, 421-433.

Corsi, F., De Leeuw, J., \& Skidmore, A. (2000). Modelling species distribution with GIS In L. Boitani \& K. Fuller (Eds.), Research techniques in animal ecology: controversies and consequences (389-434 pp). New York, Estados Unidos: Columbia University Press.

Cushman, S. A. (2006). Effects of habitat loss and fragmentation on amphibians: a review and prospectus. Biological Conservation, 128, 231-240. doi.org/10.1016/j. biocon.2005.09.031

de la Estrella, M., Mateo, R. G., Wieringa, J.J., Mackinder, B., \& Muñoz, J. (2012). Legume diversity patterns in West Central Africa: influence of species biology on distribution models. PLOS ONE, 7, e41526. doi:10.1371/journal.pone.0041526

Dhillion, S. S., Aguilar-Støen, M., \& Camargo-Ricalde, S. L. (2004). Integrative ecological restoration and local involvement in the Tehuacán-Cuicatlán Valley, Mexico. Environmental Conservation, 13, 1-3. doi:10.1017/S0376892904001043
Elith, J. C. H, Anderson, R. P., Dudík, M., Ferrier, S., Guisan, A., Hijmans, R. J., Huettmann, M., Leathwick, J. R., Lehmann, A., Lohmann, L. G., Lois, B. A., Manion, G., Moritz, C., Nakamura, M., Overton, J. M., Peterson, T., Phillips, S. J., Richardson, K., Scachetti-Pereira, P., Schapire, R. E., Soberón, J., Williams, S., Wisz, M. S., \& Zimmermann, N. (2006). Novel methods improve prediction of species' distributions from occurrence data. Ecography, 29, 129-151. doi: 10.1111/j.2006.0906-7590.04596.x

Engler, R., Guisan, A., \& Rechsteiner, L. (2004). An improved approach for predicting the distribution of rare and endangered species from occurrence and pseudo-absence data. Journal of Applied Ecology, 41, 263-274. doi: 10.1111/j.0021-8901.2004.00881.x

Escalante, E. T. (2003). Determinación de prioridades en las áreas de conservación para los mamíferos terrestres de México, empleando criterios biogeográficos. Anales del Instituto de Biología, Serie Zoología, 74, 211-238.

ESRI. (2014). ArcMap, versión 10.2.2. Redlands, CA: ESRI (Environmental Systems Resource Institute).

García, E.-Comisión Nacional para el Conocimiento y Uso de la Biodiversidad (CONABIO). (1998). 'Climas' (clasificación de Koppen, modificado por García). Escala 1:1000000. México.

Gómez-Díaz, J. D., Monterroso-Rivas, A. I., \& TinocoRueda, J. A. (2007). Comportamiento de la vegetación bajo escenarios de cambio climático en la reserva de la Biosfera Barranca de Metztitlán, Hidalgo, México. Zonas Áridas, 11, 61-69. doi: http:// dx.doi.org/10.21704/za.v11i1.204

Grether, R., Camargo-Ricalde, S. L., \& Martínez-Bernal, A. (1996). Especies del género Mimosa (Leguminosae) presentes en México. Boletín de la Sociedad Botánica de México, 58, 149-152.

Grether, R., Martínez-Bernal, A., \& Camargo-Ricalde, S. L. (2007). Mimosa. In J. Rzedowski y G. Calderón de Rzedowski (Eds.), Flora del Bajio y de regiones adyacentes Fascículo 150 (140-183 pp.). México: Instituto Ecología, Centro Regional del Bajío.

Grether, R., Camargo-Ricalde, S. L., Martínez-Bernal, A., Montaño-Arias, S., \&. Fraile M. E. (2015). Diversity and geographical distribution patterns of the genus Mimosa (Mimosoideae) in the United States, Mexico, and Central America. In R. Fortunato (Ed.), $V$ Conferencia Internacional de Leguminosas (VILC). Buenos Aires, Argentina: Fundación CICCUS.

Grinnell, J. (1917). The niche-relationships of the California Thrasher. Auk, 34, 427-434. doi: 10.2307/4072271

Guevara-Escobar, A., Gonzáles-Sosa, E., Susán-Azpiri, H., Malda-Barrera, G., Martínez y Díaz, M., GómezSánchez, M., Hernández-Sandoval, L., Pantoja-Hernández, Y., \& Olvera-Valerio, D. (2008). Distribución 
potencial de algunas leguminosas arbustivas en el Altiplano Central de México. Agrociencia, 42, 703-716.

Hernández, P. A., Graham, C. H., Master, L. L., \& Albert D. L. (2006). The effect of sample size and species characteristics on performance of different species distribution modeling methods. Ecography, 29, 773785. doi: 10.1111/j.0906-7590.2006.04700.x

Herrera, C. A. (2012). Aplicación de tecnologías de información para modelar la distribución de plagas (Tesis de maestría). Universidad de Costa Rica, Costa Rica.

Hijmans, R. J., Cameron, S. E., Parra, J. L., Jones, P. G., \& Jarvis, A. (2005). Very high resolution interpolated climate surfaces for global land areas. International Journal of Climatology, 25, 1965-1978. doi: 10.1002/ joc. 1276

Hole, D. G., Willis, S. G., Pain, D. J., Fishpool, L. D., Butchart, S. H. M., Collingham, Y. C., Rahbek, C., \& Huntley, B. (2009). Projected impacts of climate change on a continent wide protected area network. Ecology Letters, 12, 420-431. doi: 10.1111/j.1461-0248.2009.01297.x

Hutchinson, G. E. (1944). Limnological studies in Connecticut. VII. A critical examination of the supposed relationship between phytoplakton periodicity and chemical changes in lake waters. Ecology, 25, 3-26. doi: $10.2307 / 1930759$

Hutchinson, G. E. (1957). A Treatise on Limnology. Volume I Geography, Physics, and Chemistry. New York, Estados Unidos: Chapman and Hall.

INEGI (Instituto Nacional de Estadística, Geografía e Informática). (2005). Catálogo de claves de entidades federativas, municipios y localidades. Recuperado de http://geoweb.inegi.org.mx/mgn2k/catalogo.jsp

INIFAP (Instituto Nacional de investigaciones Forestales y Agropecuarias)- CONABIO (Comisión Nacional para el Conocimiento y Uso de la Biodiversidad). (1995). 'Edafología'. Escalas 1:250000 y 1:1000000. México.

Jeschke, J. M., \& Strayer, D. L. (2008). Usefulness of bioclimatic models for studying climate change and invasive species. Annals of the New York Academy of Sciences, 1134, 1-24. doi: 10.1196/annals.1439.002

Kumar, S., \& Stohlgren, T. J. (2009). MAXENT modelling for predicting suitable habitat for threatened and endangered tree Canacomyrica monticola in New Caledonia. Journal of Ecology and Natural Environment, 1, 94-98.

Lindenmayer, D. B., Mackey, B. G., \& Nix, H. A. (1996). The bioclimatic domains of four species of commercially important eucalypts from south-eastern Australia. Australian Forestry, 59, 74-89. doi: http://dx.doi. org/10.1080/00049158.1996.10674672
Lobo, J. M., Jiménez-Valverde, A., \& Real, R. (2008). AUC: a misleading measure of the performance of predictive distribution models. Global Ecology and Biogeography, 17, 145-151. doi: 10.1111/j.1466-8238.2007.00358.x

Marini, M. Â., Barbet-Massin, M., Martínez, J., Prestes, N. P., \& Jiguet, F. (2010). Applying ecological niche modelling to plan conservation actions for the Red-spectacled Amazon (Amazona pretrei). Biological Conservation, 143, 102-112. doi:10.1016/j. biocon.2009.09.009

Marmion, M., Parviainen, M., Luoto, M., Heikkinen, R. K., \& Thuiller, W. (2009). Evaluation of consensus methods in predictive species distribution modelling. Diversity and Distributions, 15, 56-69. doi: 10.1111/j.1472-4642.2008.00491.x

Martínez-Bernal, A., \& Grether, R. (2006). Mimosa. In A. Novelo \& R. Medina-Lemus (Eds.), Flora del Valle de Tehuacán-Cuicatlán (42-99pp) Fascículo 44. México: Instituto de Biología, Universidad Nacional Autónoma de México.

Martínez-Bernal, A., Grether, R., \& González-Amaro, R. M. (2008). Leguminosae I. Mimosoideae: Mimosa. Flora de Veracruz. Xalapa, Veracruz, México: Instituto. Ecología A. C.

Martínez-Pérez, G., Orozco-Segovia, A., \& Mantorell, C. (2006). Efectividad de algunos tratamientos pre-germinativos para ocho especies leñosas de la Mixteca Alta Oaxaqueña con características relevantes para la restauración. Boletín de la Sociedad Botánica de México, 79, 9-20.

Mateo, R. G. (2008). Modelos predictivos de riqueza de diversidad vegetal. Comparación y optimización de métodos de modelado ecológico (Tesis de doctorado). España: Universidad Complutense de Madrid.

Maxted, N., \& Kell, S. P. (2009). Establishment of a global network for the in situ conservation of crop wild relatives: status and needs. Rome, Italy: FAO Commission on Genetic Resources for Food and Agriculture.

Mezaour, A. (2005). Filtering web documents for a thematic warehouse case study: eDot a food risk data warehouse (extended). In Intelligent information processing and web mining. Laboratoire de Recherche en Informatique (LRI). Francia: Université Paris Sud.

Naoki, K., Gómez, M. I., López, R. P., Meneses, R. I., \& Vargas, J. (2006). Comparación de modelos de distribución de especies para predecir la distribución potencial de vida silvestre en Bolivia. Ecología en Bolivia, 41, 65-78.

Ndayishimiye, J., Greve, M., Stoffelen, P., Bigendako, M. J., De Cannière, C., Svenning, J. C., \& Bogaert, J. (2012). Modelling the spatial distribution of endemic Caesalpinioideae in Central Africa, a contribution to the evaluation of actual protected areas in the region. International Journal of Biodiversity and Conservation, 4, 118-129. doi: 10.5897/IJBC11.150 
Nix, H. A. (1986). A biogeographic analysis of Australian elapid snakes. In R. Longmore (Ed.), Atlas of elapid snakes of Australia. Australia: Australia Government Publishing Service.

Pacheco, S., Malizia, L. R., \& Cayuela, L. (2010). Effects of climate change on subtropical forests of South America. Tropical Conservation Science, 3, 423-437.

Palacios, R. (2010). Efectos del cambio climático sobre la distribución de nopales (género Opuntia y Nopalea: Cactaceae) en la región central de México (Tesis de maestría). Universidad Nacional Autónoma de México, Ciudad de México, México.

Parmesan, C., \& Yohe, G. (2003). A globally coherent fingerprint of climate change impacts across natural systems. Nature, 421, 37-42. doi:10.1038/nature01286

Pavón, N. P., Ballato-Santos, J., \& Pérez-Pérez, C. (2011) Germinación y establecimiento de Mimosa aculeaticarpa var. biuncifera (Fabaceae-Mimosoideae). Revista Mexicana de Biodiversidad, 82, 653-661.

Peterson, A. T., \& Cohoon, K. P. (1999). Sensitivity of distributional prediction algorithms to geographic data completeness. Ecological modelling, 117, 159-164. doi:10.1016/S0304-3800(99)00023-X

Peterson, A. T., Egbert, S. L., Sánchez-Cordero, V., \& Price, K. P. (2000). Geographic analysis of conservation priority: endemic birds and mammals in Veracruz, Mexico. Biological Conservation, 93, 85-94. doi:10.1016/S0006-3207(99)00074-9

Peterson, A. T., Soberón, J., Pearson, R. G., Anderson, R. P., Martínez-Meyer, E., Nakamura, M., \& Araújo, M. B. (2011). Ecological niches and geographic distributions. New Jersey, Estados Unidos: Princenton University Press.

Phillips, S. J., Dudík M., \& Schapire R. E. (2004). A maximum entropy approach to species distribution modeling. In Proceedings of the twenty-first international conference on machine learning. doi: $10.1145 / 1015330.1015412$

Phillips, S. J., Anderson, R. P., \& Schapire, R. E. (2006). Maximum entropy modelling of species geographic distributions. Ecological Modelling, 190, 231-259. doi:10.1016/j.ecolmodel.2005.03.026

Phillips Trotta, S. J., Dudík, M., Elith, J., Graham, C. H., Lehmann, A., Leathwick, J., \& Ferrier, S. (2009). Sample selection bias and presence-only distribution models: implications for background and pseudoabsence data. Ecological Applications, 19, 181-197. doi: 10.1890/07-2153.1

Ramírez-Villegas, J., Khoury, C., Jarvis, A., Debouck, D. G., \& Guarino, L. (2010). A gap analysis methodology for collecting crop genepools: a case study with Phaseolus beans. PloS one, 5, e13497. doi:10.1371/ journal.pone. 0013497

Rushton, S. P., Ormerod, S. J., \& Kerby, G. (2004). New paradigms for modelling species distributions?
Journal of Applied Ecology, 41, 193-200. doi: 10.1111/j.0021-8901.2004.00903.x

Rzedowski, J. (1991). Diversidad y orígenes de la flora fanerogámica de México. Acta Botánica Mexicana, 14, 3-21.

Soberón, J., \& Peterson, A. T. (2005). Interpretation of models of fundamental ecological niches and species distributional areas. Biodiversity Informatics, 2, 110. doi:10.17161/bi.v2i0.4

Sotuyo, S., Delgado-Salinas, A., Lewis, G. P., Chase, M. W., Ferrari, L., \& Oyama K. (2010). Filogeografía del complejo Caesalpinia hintonii: (Leguminosae: Caesalpinioideae: Poincianella). Revista Mexicana de Biodiversidad, 81, 883-894.

SPSS. (2012). IBM SPSS Statistics. Recuperado de www. ibm.com.analytics/spss.

Stockwell, D., \& Peters, D. (1999). The GARP modeling system: problems and solutions to automated spatial prediction. International Journal of Geographical Information Science, 13, 143-158. doi:10.1080/136588199241391

Thiers, B. 2016 [actualización continua]. Index Herbariorum: A global directory of public herbaria and associated staff. New York Botanical Garden's virtual Herbarium. Recuperado de http://sweetgum. nybg.org/ih.

Trotta-Moreu, M. N., Lobo, J. M., \& Cabrero-Sañudo, F. J. (2008). Distribución conocida y potencial de las especies de Geotrupinae (Coleoptera: Scarabaeoidea) en México. Acta Zoológica Mexicana, 24, 39-65.

Villaseñor, J. L., \& Téllez-Valdez, O. (2004). Distribución potencial de las especies del género Jefea (Asteraceae) en México. Anales del Instituto de Biología, Serie Botánica, 75, 205-220.

Villaseñor, J. L. (2016). Checklist of the native vascular plants of Mexico. Revista Mexicana de Biodiversidad, 87, 1-344. doi:10.1016/j.rmb.2016.06.017

Wisz, M. S., Hijmans, R. J., Li, J., Peterson, A. T., Graham, C. H., \& Guisan, A. (2008). Effects of sample size on the performance of species distribution models. Diversity and Distributions, 14, 763-773. doi: $10.1111 /$ j.1472-4642.2008.00482.x

WorldClim. (s.f). Data for current conditions ( 19502000). Recuperado de http://www.worldclim.org/ current.

Yberri, F. G. (2009). Distribución geográfica de Nopalxochia phyllanthoides (DC) Britton et Rose (Cactaceae): Modelos predictivos y conservación (Tesis de licenciatura). Universidad Nacional Autónoma de México, D. F., México. 